\title{
Brain region-specific spread of Lewy body pathology in synucleinopathies is governed by a-synuclein conformations.
}

\section{Tim Bartels ( $\boldsymbol{\nabla}$ t.bartels@ucl.ac.uk)}

Dementia Research Institute, UCL

\section{Laura de Boni}

Dementia Research Institute, University College London

\section{Aurelia Hays Watson}

Dementia Research Institute, University College London

\section{Ludovica Zaccagnini}

Dementia Research Institute, University College London

Nora Kim

Department of Neurosurgery, NYU Grossman School of Medicine

John Sanderson

Ann Romney Center for Neurologic Diseases, Brigham and Women https://orcid.org/0000-0002-11682540

\section{Haiyang Jiang}

Ann Romney Center for Neurologic Diseases, Brigham and Women's Hospital and Harvard Medical School

\section{Elodie Martin}

Dementia Research Institute, University College London

\section{Adam Cantlon}

Ann Romney Center for Neurologic Diseases, Brigham and Women's Hospital and Harvard Medical School

\section{Matteo Rovere}

Ann Romney Center for Neurologic Diseases, Brigham and Women's Hospital and Harvard Medical School https://orcid.org/0000-0002-3260-4955

\section{Lei Liu}

Brigham and Women's Hospital https://orcid.org/0000-0002-4604-4629

\section{Marc Sylvester}

Core Facility Mass Spectrometry, Institute of Biochemistry and Molecular Biology, Medical Faculty, University of Bonn

\section{Tammaryn Lashley}

Queen Square Brain Bank for Neurological studies 


\section{Ulf Dettmer}

Ann Romney Center for Neurologic Diseases, Brigham and Women's Hospital and Harvard Medical School, Boston, MA 02115

\section{Zane Jaunmuktane}

Division of Neuropathology, The National Hospital for Neurology and Neurosurgery, University College London Hospitals NHS Foundation Trust, London, UK

\section{Letter}

Keywords: Parkinson's disease (PD), Dementia with Lewy Bodies (DLB), a-synuclein protein (aS)

Posted Date: March 10th, 2021

DOl: https://doi.org/10.21203/rs.3.rs-294515/v1

License: (c) (i) This work is licensed under a Creative Commons Attribution 4.0 International License. Read Full License 


\title{
Brain region-specific spread of Lewy body pathology in synucleinopathies is governed by $\alpha$-synuclein conformations.
}

\author{
Authors: \\ Laura de Boni ${ }^{1,2}$, Aurelia Hays Watson ${ }^{1}$, Ludovica Zaccagnini ${ }^{1}$, Nora Kim ${ }^{3}$, John Sanderson ${ }^{4}$, \\ Haiyang Jiang ${ }^{5}$, Elodie Martin ${ }^{1}$, Adam Cantlon $^{5}$, Matteo Rovere $^{7}$, Lei Liu ${ }^{5}$, Marc Sylvester ${ }^{7}$, \\ Tammaryn Lashley $^{8}$, Ulf Dettmer ${ }^{5}$, Zane Jaunmuktane ${ }^{8,9,10}$, Tim Bartels $^{1^{*}}$
}

\section{Affiliations:}

1 Dementia Research Institute, University College London, London, United Kingdom

2 Department of Neurology, University Hospital Bonn, Germany

3 Department of Neurosurgery, NYU Grossman School of Medicine, New York, NY, USA

4 Warren Alpert Medical School of Brown University, Providence, RI, USA

5 Ann Romney Center for Neurologic Diseases, Brigham and Women's Hospital and Harvard Medical School, Boston, MA, USA

6 Scuola Normale Superiore di Pisa, Faculty of Sciences, Pisa, Italy

7 Core Facility Mass Spectrometry, Institute of Biochemistry and Molecular Biology, Medical Faculty, University of Bonn

8 Division of Neuropathology, National Hospital for Neurology and Neurosurgery, University College London NHS Foundation Trust, Queen Square, London, UK.

9 Queen Square Brain Bank for Neurological disorders, UCL Queen Square Institute of Neurology, Queen Square, London, UK

10 Department of Clinical and Movement Neurosciences, University College London, London, UK

*Correspondence to: T.B. (email: t.bartels@ucl.ac.uk) 
The $\alpha$-synuclein protein $(\alpha S)$ is the major constituent of pathological neuronal inclusions both in Parkinson's disease (PD) and Dementia with Lewy Bodies (DLB) ${ }^{1}$ with differential brain region-specific pathology patterns and clinical presentations. Two hypotheses that were recently put forward were either that of brain-region specific vulnerability or that of different $\alpha$ S aggregates, "strains", that would affect different brain regions via "prion-like" spread. What governs these patterns, their hypothesized "prion-like" progression and region-specific vulnerability to $\alpha \mathrm{S}$ aggregation in different synucleinopathies is still largely unknown. Data collected in the last decade suggests that $\alpha \mathrm{S}$ can exhibit in different conformations under physiological and/or pathological conditions, which in turn help govern aggregation propensity. The cytosolic unfolded, monomeric form of $\alpha \mathrm{S}\left(\alpha \mathrm{S}^{\mathrm{CU}}\right)$ is aggregation-prone and can misfold into soluble, toxic oligomers, protofilaments, and amyloid fibrils with self-templating properties ${ }^{2}$, while the cytosolic helically folded, multimeric form $\left(\alpha S^{\mathrm{CH}}\right)$ resists disease-associated changes ${ }^{3,4}$.

It was demonstrated by our and other laboratories that mutations of $S N C A$ leading to familial Parkinson's disease (fPD) and various genetic PD risk factors for developing sporadic PD destabilize physiological, aggregation-resistant $\alpha \mathrm{S}^{\mathrm{CH} 3,5-10}$.

To address the question of whether the equilibrium of $\alpha \mathrm{S}^{\mathrm{CU}}$ and $\alpha \mathrm{S}^{\mathrm{CH}}$ also plays an important role in sporadic synucleinopathies, we adapted our established ${ }^{3,11,12}$ multimer assay (using crosslinking and Western blot analysis of lysate) to frozen human post-mortem tissue (Fig. S16). This protocol revealed a prominent cytosolic $\sim 80 \mathrm{kDa}$ and $14 \mathrm{kDa} \alpha \mathrm{S}$ species (Fig 1a, Fig. S2-4) as described previously ${ }^{11-13}$. The two cytosolic $\alpha \mathrm{S}$ species corresponded to a $86 \mathrm{kDa}$ helically folded homo-oligomer and a $14 \mathrm{kDa}$ monomeric form, respectively, as confirmed by Circular Dichroism (CD) spectroscopy, Multi-Angle-Light Scattering, Mass spectrometry and 
Western blotting conducted on purified $14 \mathrm{kDa}$ and $86 \mathrm{kDa}$ species from human brain tissue and HEK cells (Fig. 1b, Table S1, Fig. S5, Fig. S7-14).
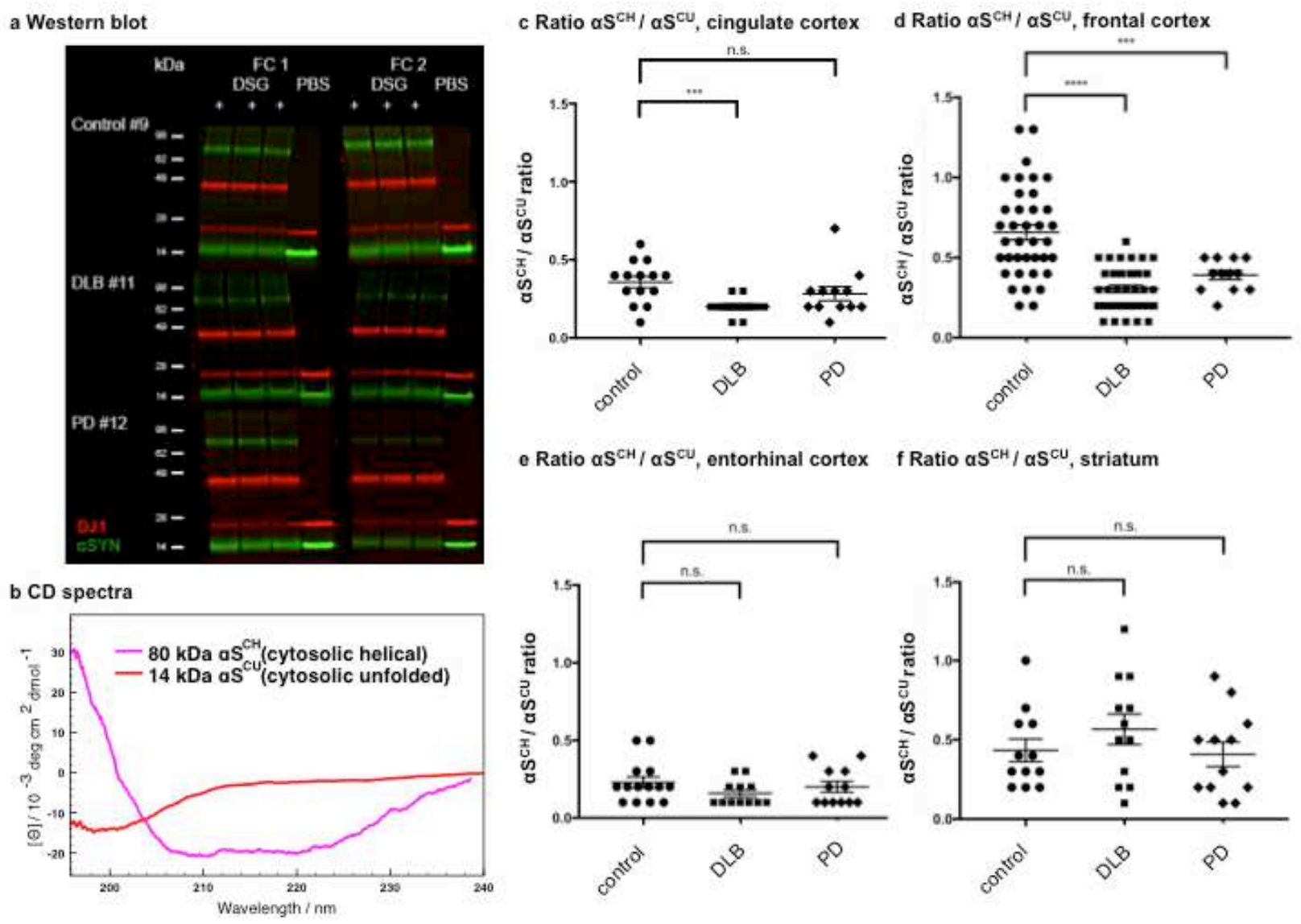

Fig. 1 Disturbed equilibria of cytosolic helically folded and unfolded physiological $\alpha S$ in PD

and DLB patients. a, Representative Western blot of $\alpha \mathrm{S}^{\mathrm{CH}}$ and $\alpha \mathrm{S}^{\mathrm{CU}}$ in controls, DLB and PD patients. Each piece of brain was analyzed in duplicates (frontal cortex FC1 and FC2). The crosslinking reaction was performed in technical triplicates alongside one non-crosslinked (PBS) sample. The Western blot demonstrates reduced $\alpha \mathrm{S}^{\mathrm{CH}}$ to $\alpha \mathrm{S}^{\mathrm{CU}}$ ratios in DLB and PD patients compared to the control. DSG "++" = crosslinked sample. DSG "_" $=$ non-crosslinked sample. Green $=\alpha \mathrm{S}$, red $=\mathrm{DJ} 1 . \mathbf{b}, \mathrm{CD}$ spectroscopy of immunoprecipitated and separated (size-exclusion chromatography) $\alpha \mathrm{S}^{\mathrm{CH}}$ and $\alpha \mathrm{S}^{\mathrm{CU}}$ from human frontal cortex control brain tissue. The $\alpha \mathrm{S}$ 
multimer from human brain exhibits an $\alpha$-helical secondary structure of approximately $48 \%$. c, Significant reduction of $\alpha \mathrm{S}^{\mathrm{CH}} / \alpha \mathrm{S}^{\mathrm{CU}}$ ratio in the cingulate cortex comparing controls $(\mathrm{n}=7)$ and DLB $(n=7)$ patients. No significant alteration of the $\alpha \mathrm{S}^{\mathrm{CH}} / \alpha \mathrm{S}^{\mathrm{CU}}$ equilibria in the cingulate cortex comparing controls $(n=7)$ and $\mathrm{PD}(n=6)$ patients. d, Significant reduction of $\alpha \mathrm{S}^{\mathrm{CH}} / \alpha \mathrm{S}^{\mathrm{CU}}$ ratio in the frontal cortex comparing controls $(n=19)$ to PD $(n=6)$ and DLB $(n=19)$ patients. e, No significant alteration of the $\alpha \mathrm{S}^{\mathrm{CH}} / \alpha \mathrm{S}^{\mathrm{CU}}$ equilibria in the entorhinal cortex comparing controls $(\mathrm{n}=$ 7) to $\mathrm{PD}(\mathrm{n}=6)$ and $\mathrm{DLB}(\mathrm{n}=6)$ patients. f, No significant alteration of the $\alpha \mathrm{S}^{\mathrm{CH}} / \alpha \mathrm{S}^{\mathrm{CU}}$ equilibria in the striatum comparing controls $(n=6)$ to PD $(n=6)$ and DLB $(n=6)$ patients.

Next, we assessed the ratio of cytosolic helical and unfolded $\alpha \mathrm{S}\left(\alpha \mathrm{S}^{\mathrm{CH}} / \alpha \mathrm{S}^{\mathrm{CU}}\right)$ in four different brain regions (entorhinal cortex (EC), cingulate cortex (CC), frontal cortex (FC) and striatum) of 28 age-matched individuals, classified as neurological controls, 22 DLB patients and 13 sporadic PD patients (Fig. S15-16). We found that DLB patients exhibited a significant reduction of $\alpha \mathrm{S}^{\mathrm{CH}} / \alpha \mathrm{S}^{\mathrm{CU}}$ in the CC $(\mathrm{p}=0.0007$, Fig. 1d) and $\mathrm{FC}(\mathrm{p}<0.0001$, Fig. 1e). PD patients exhibited a significant decrease in the FC ( $p<0.001$, Fig. 1d). We did not observe significant changes between patients and controls in the EC, a region typically affected by Lewy pathology earlier in the disease course than neocortical regions (Fig. 1a), the striatum which served as a control region (due to low Lewy body (LB) burden, Fig. 1e) or the internal control protein DJ1 (Fig. S17). The evaluation of these findings with regard to clinical and neuropathological features (summary Fig. S15) revealed a significant negative correlation between the reduction of the $\alpha \mathrm{S}^{\mathrm{CH}} / \alpha \mathrm{S}^{\mathrm{CU}}$ ratio in $\mathrm{FC}$ or $\mathrm{CC}$ and $\alpha \mathrm{S}$ Braak LB staging $(\mathrm{FC} r=-0.6, \mathrm{p}<0.0001 ; \mathrm{CC} r=-0.4, \mathrm{p}=$ $0.006)$ or McKeith staging ( $\mathrm{FC} r=-0.6, \mathrm{p}<0.0001 ; \mathrm{CC} \mathrm{r}=-0.4, \mathrm{p}=0.008$, Fig. 2a-d). 


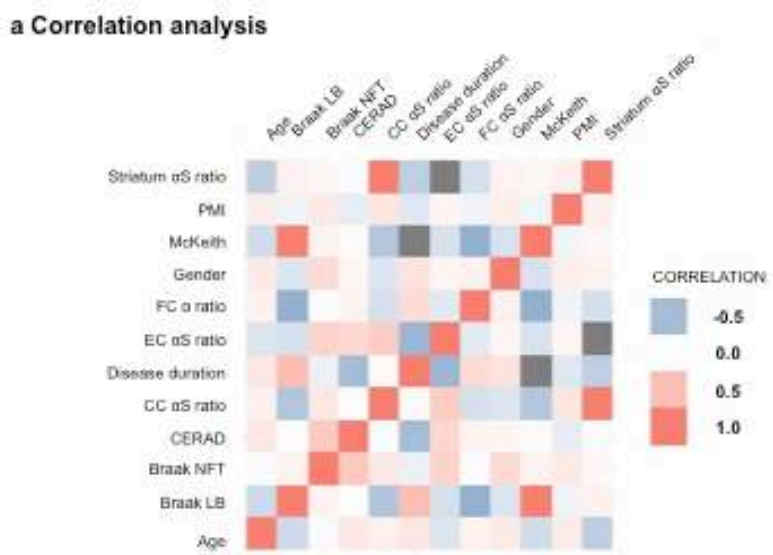

\begin{tabular}{|c|c|c|c|c|c|}
\hline & r & $\begin{array}{c}95 \% \\
\text { oonfidence } \\
\text { interval }\end{array}$ & $R^{2}$ & $\begin{array}{c}\text { p- } \\
\text { value }\end{array}$ & $\begin{array}{c}\text { number } \\
\text { pairs }\end{array}$ \\
\hline $\begin{array}{l}\text { FC as } \mathrm{S}^{\mathrm{CH}} / \text { as } \mathrm{Cu} \text { ratio vs. } \\
\text { McKaith staghg }\end{array}$ & -0.6 & $\begin{array}{c}-0.7223 \text { to } \\
-0.4394\end{array}$ & 0.4 & $=0.0001$ & 82 \\
\hline $\begin{array}{c}\mathrm{FC} \alpha 5^{\mathrm{CH} /} / \alpha \mathrm{S}^{\mathrm{CU}} \text { ratio vs. } \\
\text { Braak LB staging }\end{array}$ & -0.6 & $\begin{array}{c}-0.7223 \text { to } \\
-0.4393\end{array}$ & 0.4 & $\angle 0.0001$ & 82 \\
\hline $\begin{array}{c}\text { CC aSci/ ascu ratio vs. } \\
\text { Mckeith staging }\end{array}$ & -0.4 & $\begin{array}{c}-0.6496 \text { to } \\
-0.1207\end{array}$ & 02 & 0.008 & 39 \\
\hline $\begin{array}{c}\mathrm{CC} \text { as } \mathrm{CH} / \text { aS } \mathrm{Cu} \text { ratio v8. } \\
\text { Brabik LB staging }\end{array}$ & -0.4 & $\begin{array}{c}-0.6584 \text { to } \\
-0.1367\end{array}$ & 0.2 & 0.005 & 39 \\
\hline
\end{tabular}
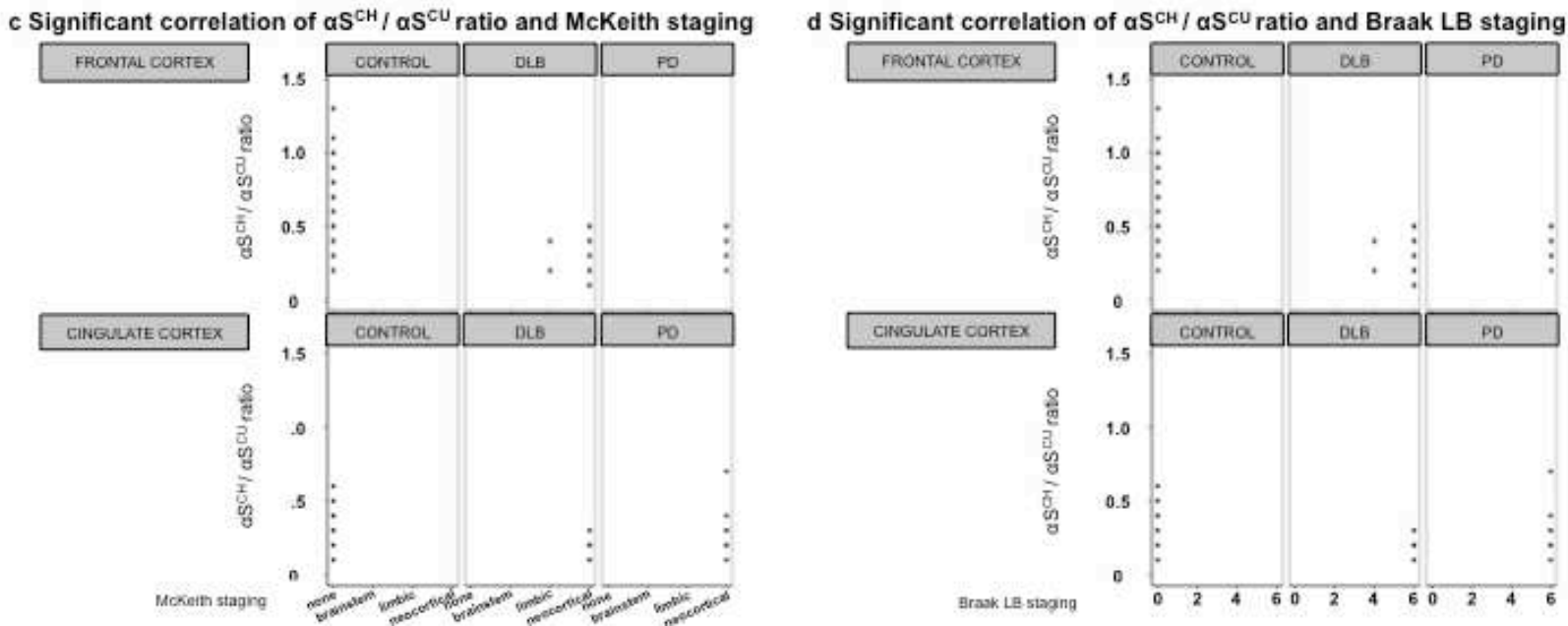

Fig. 2 Decreased $\alpha S^{\mathrm{CH}} / \alpha \mathrm{S}^{\mathrm{CU}}$ ratios correlate with LB pathology. a, Heatmap of Pearson correlation coefficients. Controls were set to 0 for McKeith and Braak LB (LB) staging. b, Significant correlation (Pearson correlation coefficient) of decreased $\alpha \mathrm{S}^{\mathrm{CH}} / \alpha \mathrm{S}^{\mathrm{CU}}$ ratios and McKeith or LB staging in the frontal and cingulate cortex, respectively. c, Graph of correlated samples $\left(\alpha \mathrm{S}^{\mathrm{CH}} / \alpha \mathrm{S}^{\mathrm{CU}}\right.$ ratios vs. McKeith). d, Graph of correlated samples $\left(\alpha \mathrm{S}^{\mathrm{CH}} / \alpha \mathrm{S}^{\mathrm{CU}}\right.$ vs. Braak LB). $\mathrm{LB}=$ Lewy body, NFT = neurofibrillary tangle, $\mathrm{CERAD}=$ Consortium to Establish a Registry for Alzheimer's Disease, $\mathrm{CC}=$ cingulate cortex, $\mathrm{EC}=$ entorhinal cortex, $\mathrm{FC}=$ frontal cortex, $\mathrm{PMI}=$ post-mortem interval. 
We did not detect a significant correlation between the altered $\alpha \mathrm{S}^{\mathrm{CH}} / \alpha \mathrm{S}^{\mathrm{CU}}$ ratio and the Braak or McKeith staging in the EC or the striatum (Fig. S18). A significant negative correlation exists for disease duration and reduction of $\alpha \mathrm{S}^{\mathrm{CH}} / \alpha \mathrm{S}^{\mathrm{CU}}$ in the EC (Fig. S19), a region being early affected in the course of the disease. Gender, age, post-mortem interval and the extend of Alzheimer's disease type neuropathological stages were not associated with the reduction of $\alpha \mathrm{S}^{\mathrm{CH}} / \alpha \mathrm{S}^{\mathrm{CU}}$ (Fig. S20-24). Biochemical analysis via detergent fractionation and ELISA of PD patient tissue ${ }^{14}$ exhibited significantly higher levels of pathology-associated, insoluble $\alpha \mathrm{S}$ in early affected brain regions (EC and CC) according to the classical caudal-rostral progression (Fig. S25) matching the measured relative destabilization of $\alpha \mathrm{S}^{\mathrm{CH}}$ in these brain regions. DLB patients exhibiting early cortical involvement display higher levels of pathological, insoluble $\alpha \mathrm{S}$ in the FC (Fig. S25).

Given the apparent region specificity of the $\alpha \mathrm{S}^{\mathrm{CH}}$ form, and the discussed "prion-like" spread of pathological $\alpha \mathrm{S}$ in a highly region-specific manner, we next evaluated the effect of $\alpha \mathrm{S}^{\mathrm{CH}} / \alpha \mathrm{S}^{\mathrm{CU}}$ ratios on the susceptibility of a cell towards "prion-like" fibrillar $\alpha \mathrm{S}\left(\alpha \mathrm{S}^{\mathrm{F}}\right)$. To determine the "prion-like" aggregation resistance of $\alpha S^{\mathrm{CH}}$, we performed a Thioflavin T-based aggregation assay on purified and separated $\alpha \mathrm{S}^{\mathrm{CH}}$ multimers and $\alpha \mathrm{S}^{\mathrm{CU}}$ monomers. On native non-crosslinked $\alpha \mathrm{S}^{\mathrm{CH}}$ (isolated from human erythrocytes) our assay showed that the $\alpha \mathrm{S}^{\mathrm{CH}}$-in contrast to monomeric $\alpha \mathrm{S}^{\mathrm{CU}}$ - did not form ThT-bound fibrils (Fig. 3a). The addition of recombinant "prionlike" $\alpha S^{\mathrm{F}}$ did stimulate the aggregation in the $\alpha S^{\mathrm{CU}}$ monomer but not in the $\alpha \mathrm{S}^{\mathrm{CH}}$ multimer, hence indicating that the cytosolic helical structure found in human brain is not susceptible to putative 
"prion-like" spread. We examined the "prion-like" aggregation susceptibility of the neuroblastoma $\alpha \mathrm{S}$ fPD M17D cells by adding $\alpha \mathrm{S}^{\mathrm{F}}$ to the growth medium and assessed the amount of (Triton-X) insoluble $\alpha \mathrm{S}$ found in the cell pellets by $\alpha \mathrm{S}$ ELISA after 2 days of seeding. While no differences in forming insoluble $\alpha \mathrm{S}$ was found in cells expressing fPD mutations without treatment (Fig. 3b), the addition of $\alpha \mathrm{S}^{\mathrm{F}}$ to the growth medium led to a significant increase of aggregated $\alpha \mathrm{S}$ across all fPD mutations, indicating an increased "prion-like" aggregation susceptibility compared to wild type (wt, Fig. 3c). This directly indicates that all fPD mutations lead to greater susceptibility towards "prion-like" aggregation in neuronal cells while their effect on spontaneous aggregation is comparatively small. In accordance with previous published data ${ }^{5}$, we detected a decrease of $\alpha \mathrm{S}^{\mathrm{CH}} / \alpha \mathrm{S}^{\mathrm{CU}}$ in the lysate of M17D cells expressing different familial PD SNCA mutations (A30P, G51D, A53T, E46K and H50Q, respectively) compared to wt (Fig. 3d). The decrease of $\alpha \mathrm{S}^{\mathrm{CH}} / \alpha \mathrm{S}^{\mathrm{CU}}$ in these mutants correlated significantly with the susceptibility towards "prion-like" aggregation as measured by insoluble $\alpha \mathrm{S}$ in $\alpha \mathrm{S}^{\mathrm{F}}$ seeded M17D cells ( $p=0.002$, Fig. $3 d$ ). In contrast, M17D cells with higher expression ratios of $\alpha \mathrm{S}^{\mathrm{CH}} / \alpha \mathrm{S}^{\mathrm{CU}}$ exhibited decreased seeding and aggregation capabilities, indicating that increased $\alpha \mathrm{S}^{\mathrm{CH}}$ stabilization in neuronal cells is protective against "prion-like" propagation (Fig. 3d). 
a ThT-aggregation assay

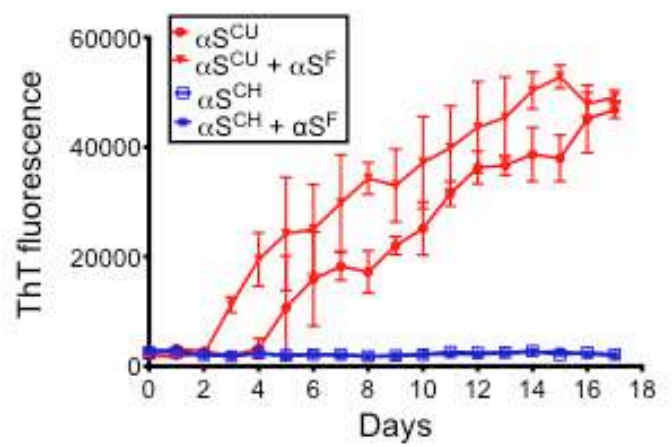

c Amounts of insouble aS in aS $^{\mathrm{F}}$ seeded M17D fPD

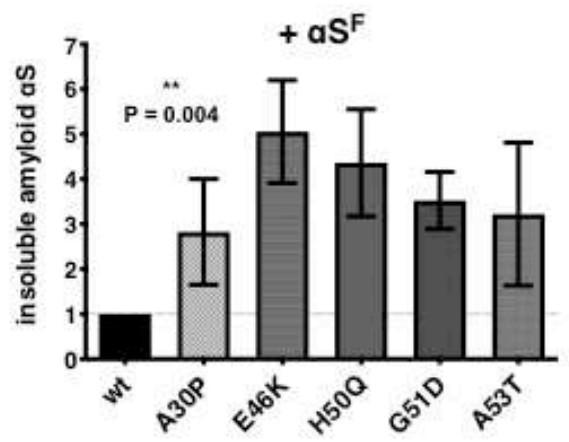

b Amounts of insouble aS in M17D fPD

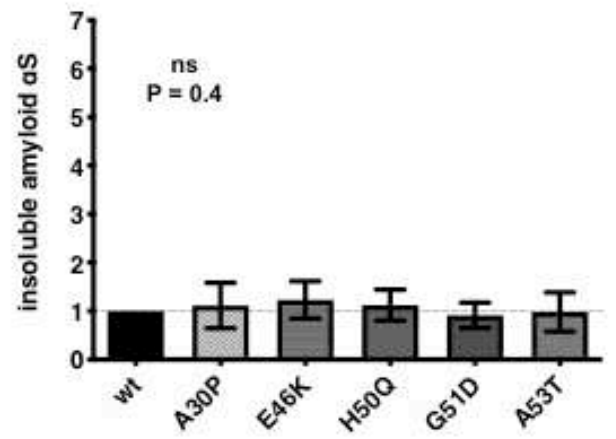

d Correlation of $\alpha \mathrm{S}^{\mathrm{CH} /} \alpha \mathrm{S}^{\mathrm{cu}}$ ratio and insoluble $\alpha \mathrm{S}$ in M17D fPD

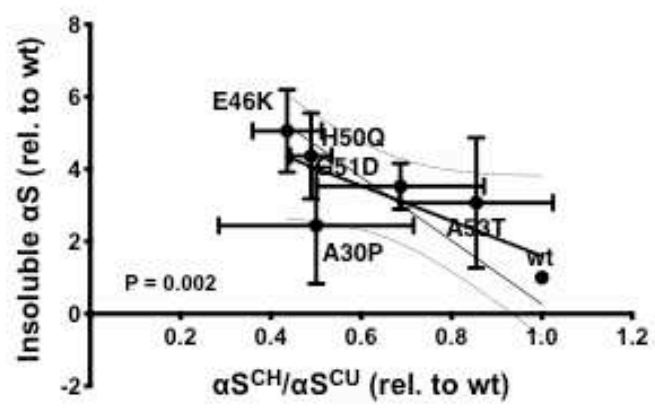

Fig. $3 \alpha \mathrm{S}^{\mathrm{CH}}$ is resistant to spontaneous or "prion-like" induced aggregation compared to $\alpha S^{\mathrm{CU}}$ and modulates "prion-like" propagation susceptibility in cellular models of disease. a, Thioflavin T fluorescence assay monitoring the aggregation of recombinant $\alpha \mathrm{S}^{\mathrm{CU}}$ and purified $\alpha \mathrm{S}^{\mathrm{CH}}$ from erythrocytes. Samples were $100 \mu \mathrm{M} \alpha \mathrm{S}$ protein each, nutated at $37{ }^{\circ} \mathrm{C}$. In samples that were seeded, $10 \mathrm{nM}$ recombinant fibrillar $\alpha \mathrm{S}\left(\alpha \mathrm{S}^{\mathrm{F}}\right)$ (i.e., 1:1000) was added. The $\alpha \mathrm{S}^{\mathrm{CH}}$ multimer demonstrated resistance to both spontaneous and seeded aggregation. $\mathbf{b}$, M17D cells transfected with wild type $\alpha \mathrm{S}$, or the fPD related mutations A30P, E46K, H50Q, G51D and A53T. Cells with fPD mutations display equal amounts of insoluble $\alpha \mathrm{S}$. c, $\alpha \mathrm{S}^{\mathrm{F}}$-seeded M17D cells display increased levels of insoluble $\alpha \mathrm{S}$. d, $\alpha \mathrm{S}^{\mathrm{CH}}$ destabilization correlates significantly (Deeming 
regression analysis) with susceptibility towards $\alpha \mathrm{S}$ aggregation as measured by insoluble $\alpha \mathrm{S}$ in M17D after 2 days of seeding $(\mathrm{P}=0.002)$. Cells were analyzed in biological triplicates.

To validate the association between the region-specific distribution and the proposed "prionlike" spreading theory of $\alpha \mathrm{S}$, we carried out an analysis of 9 brain regions from 3 DLB and 3 sporadic PD patients with advanced neocortical Lewy pathology corresponding to Braak stage 6 (Table S2). The brain regions selected for the analysis reflect the typical temporal development of LB pathology across the limbic and neocortical regions (Fig. 4). In all of these regions, control patients showed equal $\alpha \mathrm{S}^{\mathrm{CH}} / \alpha \mathrm{S}^{\mathrm{CU}}$ ratios. However, $\alpha \mathrm{S}^{\mathrm{CH}} / \alpha \mathrm{S}^{\mathrm{CU}}$ ratios in PD patients were decreased in early affected and increased in later affected regions resulting in a positive slope (increasing trendline calculated across all 9 brain regions) of $\alpha \mathrm{S}^{\mathrm{CH}} / \alpha \mathrm{S}^{\mathrm{CU}}$ ratios (Fig. 4). Interestingly, this pattern in the same anatomical regions was opposite for DLB patients, indicating that the "path" laid out by local $\alpha \mathrm{S}^{\mathrm{CH}} / \alpha \mathrm{S}$ CU destabilization would lead to faster progression from the brain stem towards the cortices. In summary, when the equilibrium of $\alpha \mathrm{S}^{\mathrm{CH}} / \alpha \mathrm{S}^{\mathrm{CU}}$ is disturbed towards the aggregation-prone $\alpha \mathrm{S}^{\mathrm{CU}}$, our data demonstrates that the likelihood of fibril formation, and subsequently LB inclusions increases. 


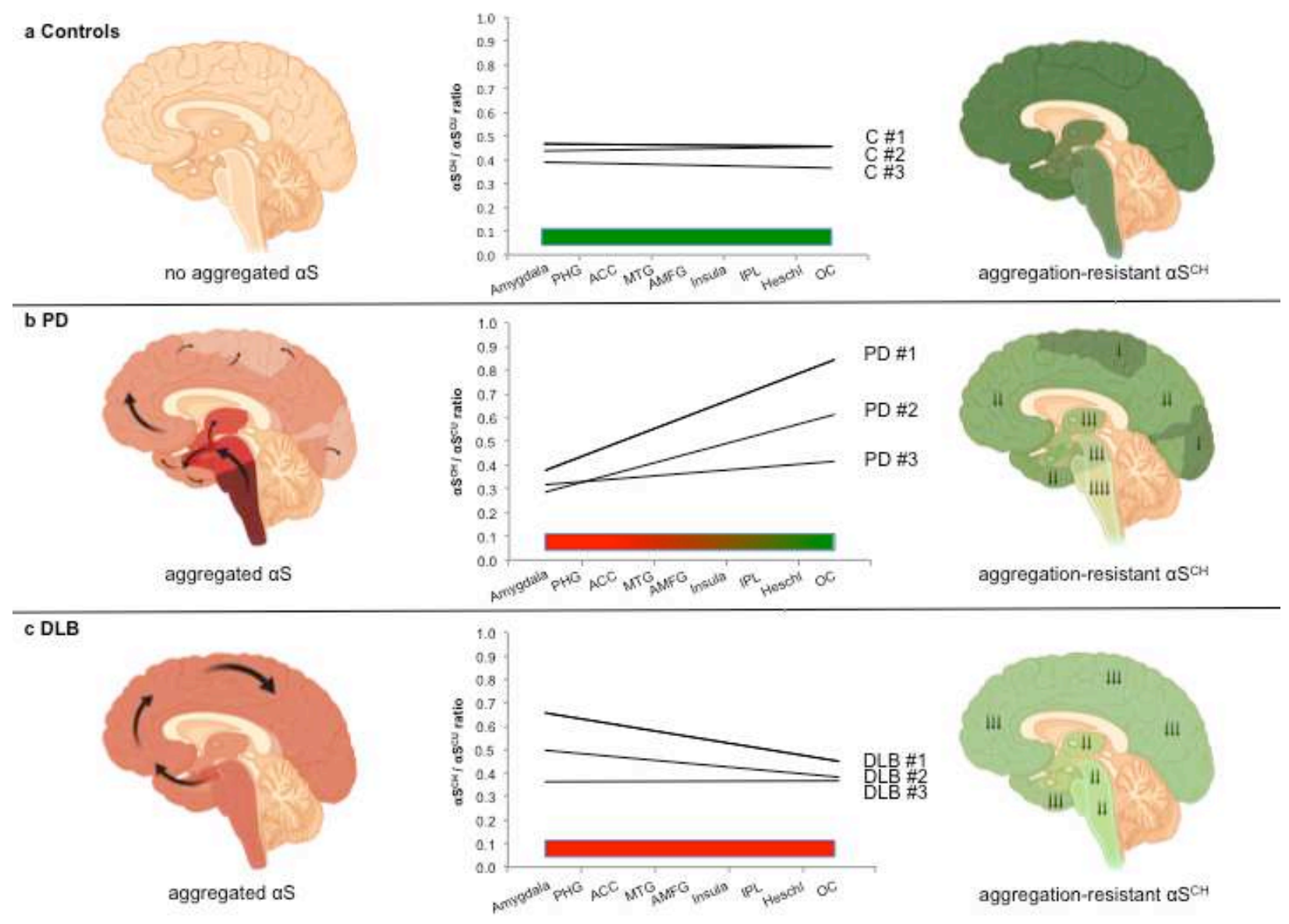

Fig. $4 \alpha S^{C H} / \alpha S^{\mathrm{CU}}$ equilibrium is disturbed in PD and DLB patients suggestive of spreading

$\boldsymbol{\alpha S}$ pathology. For each individual, 9 different brain regions were analyzed, reflecting the temporal development of LB pathology across the limbic and neocortical regions. Amygdala, cortex of the parahippocampal (PHG) and anterior cingulate gyri (ACG) are affected earlier in the disease course, followed by cortex of the insula, middle temporal (MTG), anterior middle frontal gyri (AMFG), and lastly with involvement of the cortex of inferior parietal lobule (IPL). Heschl's gyrus (Heschl) and cortex of the occipital lobe (OC) are typically spared from LB pathology in PD or involved late in the disease course. Each brain region has been analyzed in biological and technical duplicates and one non-crosslinked control sample. The linear trendlines (slopes) across all 9 brain regions is depicted for each individual. a, In controls, there is a 
balanced level of physiological $\alpha \mathrm{S}^{\mathrm{CH}} / \alpha \mathrm{S}^{\mathrm{CU}}$. b, PD patients (Braak 6) exhibit lower $\alpha \mathrm{S}^{\mathrm{CH}} / \alpha \mathrm{S}^{\mathrm{CU}}$ ratios in early affected regions according to the classical Braak staging. c, DLB patients exhibit especially low $\alpha \mathrm{S}^{\mathrm{CH}} / \alpha \mathrm{S}^{\mathrm{CU}}$ ratios in later affected, neocortical regions, including Heschl's gyrus and OC. Note that the relative measured $\alpha \mathrm{S}^{\mathrm{CH}} / \alpha \mathrm{S}^{\mathrm{CU}}$ levels, can be lower in individual control cases compared to patients even though the difference is on average reversed. The schematics on the left and right side describe the proposed model for the disequilibria of aggregated $\alpha \mathrm{S}$ and physiological, aggregation-resistant $\alpha \mathrm{S}^{\mathrm{CH}}$.

Our study demonstrated that (i) $\alpha \mathrm{S}^{\mathrm{CH}}$ multimers are present in human post-mortem brain tissue and exhibit a physiological, helical secondary structure, making them resistant to spontaneous as well as "prion-like" amyloid aggregation. (ii) The disequilibrium of $\alpha \mathrm{S}^{\mathrm{CH}}$ and $\alpha \mathrm{S}^{\mathrm{CU}}$ is brain region-specific and associated with the spreading theory of $\alpha \mathrm{S}$. In PD, it reflects the regional spread of LB pathology as proposed by Braak ${ }^{15}$. In contrast, DLB patients exhibit especially low $\alpha \mathrm{S}^{\mathrm{CH}} / \alpha \mathrm{S}^{\mathrm{CU}}$ ratios in the neocortical regions. These results are in line with current and previous analyses in familial $\mathrm{PD}$ of in vitro and in vivo models on $\alpha \mathrm{S}^{\mathrm{CH}}$, especially the helical $\alpha \mathrm{S}$ tetramer ${ }^{3,4,16,17}$. The disequilibrium of all $\alpha \mathrm{S}^{\mathrm{CH}}$ species in $\mathrm{PPD}$ models (putative $60 \mathrm{kDa}, 80 \mathrm{kDa}$ and $100 \mathrm{kDa}$ after SDS-PAGE) has previously been demonstrated ${ }^{3,5,8,18}$. Our study extends the current knowledge on $\alpha \mathrm{S}^{\mathrm{CH}}$, demonstrating the importance of the $\alpha \mathrm{S}^{\mathrm{CH}} / \alpha \mathrm{S}^{\mathrm{CU}}$ equilibrium in healthy human brain tissue and the region-specific disturbance in sporadic PD and DLB patients. Crucially, it provides a potential explanation for region-specific LB pathology in both PD and DLB. Along with these findings, our results indicate that the absolute amount of $\alpha \mathrm{S}$ might not be detrimental per se. Rather, the equilibrium of physiological aggregation-resistant and aggregation-prone forms of the $\alpha \mathrm{S}$ protein is important. Even though our in vitro data here and a 
recently published in vivo model ${ }^{18}$ indicate a causal role for $\alpha \mathrm{S}^{\mathrm{CH}}$ destabilization in neurodegeneration, it remains unclear whether the shift in the equilibrium of $\alpha \mathrm{S}^{\mathrm{CH}}$ and $\alpha \mathrm{S}^{\mathrm{CU}}$ is a primary or secondary event in synucleinopathies, or can be either. $\alpha \mathrm{S}^{\mathrm{CH}}$ destabilization could possibly result in different physiological and pathological $\alpha \mathrm{S}$ conformations in the human patient. In addition, these $\alpha$ S conformations could differentially impact neuronal vulnerability by $\alpha \mathrm{S}^{\mathrm{CH}}$ destabilization in a region-specific manner ${ }^{19-21}$. Therefore, we would reconcile the question whether local neuronal vulnerability or "prion-like" aggregation spreading explains disease progression patterns ${ }^{22}$. The answer, implied here by our data, would be that both is the case, since "prion-like" aggregation spread gets directed by local vulnerabilities. Our study focused on limbic and neocortical regions in patients with DLB and PD with most advanced Braak stage 6 neocortical LB pathology. Non-classical PD patients, in which the LB pathology does not follow the classical Braak LB staging, might not show the same disequilibria of $\alpha \mathrm{S}^{\mathrm{CH}} / \alpha \mathrm{S}^{\mathrm{CU}}$. We also did not present data on early affected brain regions, such as nucleus coeruleus, nucleus vagus or substantia nigra, given the low availability of these tissues. Still, our current findings provide a novel mechanism, in which the equilibrium of physiological aggregation-resistant $\alpha \mathrm{S}^{\mathrm{CH}}$ and physiological, aggregation-prone $\alpha \mathrm{S}^{\mathrm{CU}}$ is disturbed in sporadic PD and DLB patients. We propose that a stabilization of physiological aggregation-resistant $\alpha \mathrm{S}^{\mathrm{CH}}$ in PD and DLB patients may be beneficial in slowing down the process of neurodegeneration, analogous to efforts towards stabilizing transthyretin in familial amyloid polyneuropathy ${ }^{23,24}$. Beyond, the ratio of $\alpha \mathrm{S}^{\mathrm{CH}} / \alpha \mathrm{S}^{\mathrm{CU}}$ can be used as a biomarker of disease progression. 


\section{References and Notes:}

1. Sherer, T. B., Betarbet, R. \& Greenamyre, J. T. Pathogenesis of Parkinson's disease. Curr. Opin. Investig. Drugs 2, 657-662 (2001).

2. Meade, R. M., Fairlie, D. P. \& Mason, J. M. Alpha-synuclein structure and Parkinson's disease - lessons and emerging principles. Mol. Neurodegener. 14, 1-14 (2019).

3. Bartels, T., Choi, J. G. \& Selkoe, D. J. alpha-Synuclein occurs physiologically as a helically folded tetramer that resists aggregation. Nature 477, 107-110 (2011).

4. Wang, W. et al. A soluble alpha-synuclein construct forms a dynamic tetramer. Proc. Natl. Acad. Sci. 108, 17797-17802 (2011).

5. Dettmer, U. et al. Parkinson-causing a-synuclein missense mutations shift native tetramers to monomers as amechanism for disease initiation. Nat. Commun. 6, 1-15 (2015).

6. Dettmer, U. et al. Loss of native alpha-synuclein multimerization by strategically mutating its amphipathic helix causes abnormal vesicle interactions in neuronal cells. Hum. Mol. Genet. 26, 3466-3481 (2017).

7. Dettmer, U. et al. KTKEGV repeat motifs are key mediators of normal $\alpha$-synuclein tetramerization : Their mutation causes excess monomers and neurotoxicity. Proc Natl Acad Sci U S A. 112, 9596-9601 (2015).

8. Kim, S. et al. GBA1 deficiency negatively affects physiological alpha-synuclein tetramers and related multimers. Proc. Natl. Acad. Sci. U. S. A. 115, 798-803 (2018).

9. Iljina, M. et al. Kinetic model of the aggregation of alpha-synuclein provides insights into 
prion-like spreading. Proc. Natl. Acad. Sci. 113, E1206-E1215 (2016).

10. Burre, J., Sharma, M. \& Sudhof, T. C. alpha-Synuclein assembles into higher-order multimers upon membrane binding to promote SNARE complex formation. Proc. Natl. Acad. Sci. U. S. A. 111, E4274-83 (2014).

11. Dettmer, U., Newman, A. J., Luth, E. S., Bartels, T. \& Selkoe, D. In vivo cross-linking reveals principally oligomeric forms of $\alpha$-synuclein and $\beta$-synuclein in neurons and nonneural cells. J. Biol. Chem. 288, 6371-6385 (2013).

12. Imberdis, T., Fanning, S., Newman, A., Ramalingam, N. \& Dettmer, U. Studying $\alpha-$ Synuclein Conformation by Intact-Cell Cross-Linking. in Alpha-Synuclein: Methods and Protocols (ed. Bartels, T.) 77-91 (Springer New York, 2019). doi:10.1007/978-1-49399124-2_8

13. Luth, E. S., Bartels, T., Dettmer, U., Kim, N. C. \& Selkoe, D. J. Purification of $\alpha-$ synuclein from human brain reveals an instability of endogenous multimers as the protein approaches purity. Biochemistry 54, 279-292 (2015).

14. Sanderson, J. B. et al. Analysis of $\alpha$-synuclein species enriched from cerebral cortex of humans with sporadic dementia with Lewy bodies. Brain Commun. 2, fcaa010 (2020).

15. Braak, H. et al. Staging of brain pathology related to sporadic Parkinson's disease. Neurobiol. Aging 24, 197-211 (2003).

16. Xu, L., Bhattacharya, S. \& Thompson, D. On the ubiquity of helical alpha-synuclein tetramers. Phys. Chem. Chem. Phys. 21, 12036-12043 (2019). 
17. Gurry, T. et al. The dynamic structure of alpha-synuclein multimers. J. Am. Chem. Soc. 135, 3865-3872 (2013).

18. Nuber, S. et al. Abrogating Native $\alpha$-Synuclein Tetramers in Mice Causes a L-DOPAResponsive Motor Syndrome Closely Resembling Parkinson's Disease. Neuron 100, 7590.e5 (2018).

19. Alegre-Abarrategui, J. et al. Selective vulnerability in $\alpha$-synucleinopathies. Acta Neuropathol. 138, 681-704 (2019).

20. Jaunmuktane, Z. \& Brandner, S. On the journey to uncover the causes of selective cellular and regional vulnerability in neurodegeneration. Acta Neuropathol. 138, 677-680 (2019).

21. Engelender, S. \& Isacson, O. The Threshold Theory for Parkinson's Disease. Trends Neurosci. 40, 4-14 (2017).

22. Walsh, D. M. \& Selkoe, D. J. A critical appraisal of the pathogenic protein spread hypothesis of neurodegeneration. Nat. Rev. Neurosci. 17, 251-260 (2016).

23. Maurer, M. S. et al. Tafamidis Treatment for Patients with Transthyretin Amyloid Cardiomyopathy. N. Engl. J. Med. 379, 1007-1016 (2018).

24. Coelho L. F.; Martins da Silva, A.; Waddington Cruz, M.; Plante-Bordeneuve, V.; Lozeron, P.; Suhr, O. B.; Campistol, J. M.; Conceicao, I. M.; Schmidt, H. H.; Trigo, P.; Kelly, J. W.; Labaudiniere, R.; Chan, J.; Packman, J.; Wilson, A.; Grogan, D. R., T. . M. Tafamidis for transthyretin familial amyloid polyneuropathy: a randomized, controlled trial. Neurology 79, 785-792 (2012). 
Acknowledgments: We thank our colleagues at the Ann Romney Center for Neurologic Diseases and Department of Pathology in Boston, the Dementia Research Institute @ UCL and the Department for Neurology in Bonn for many helpful discussions.

Funding: This work was supported by grants from the UK Dementia Research Institute (DRI), which receives its funding from DRI Ltd., the UK Medical Research Council and Alzheimer's Society, and Alzheimer's Research UK (to TB); the National Institute of Neurological Disorders and Stroke NIH grants (U54-NS110435, R01-NS109209, and R21-NS107950 to TB); the Michael J. Fox Foundation (Ken Griffin Imaging Award to TB); a Parkinson's Disease Foundation Stanley Fahn Award (PF-JFA-1884 to TB); the Eisai Pharmaceutical postdoctoral programme to TB; and the Chan Zuckerberg Collaborative Pairs Initiative (to TB) and the Thiemann Foundation (to L. d. B.). Author contributions: L. d. B. and Z. J. sample curation; L. d. B., A. H. W., E. M., Z. J., L. Z., J. S., H. J., M. R., L. L., M. S., M. F., T. L., U. D. and T. B. data curation; L. d. B. and T. B. formal analysis; L. d. B., A. H. W., E. M., Z. J., L. Z., J. S., H. J., M. R., L. L., M. S., M. F., T. L., U. D. and T. B. investigation; L. d. B. writing-original draft; A. H. W., E. M., Z. J., L. Z., J. S., H. J., M. R., L. L., M. S., M. F., T. L., U. D. and T. B writingreview and editing; L. d. B., Z. J. and T. B. conceptualization; T. B. supervision; L. d. B. and T. B. funding acquisition. Competing interests: Authors declare no competing interests. Data and materials availability: All data is available in the main text or the supplementary materials.

\section{Supplementary Materials:}

Materials and Methods

Figs. S1 to S25

Table S1 and Table S2 


\section{Figures}

a Western blot

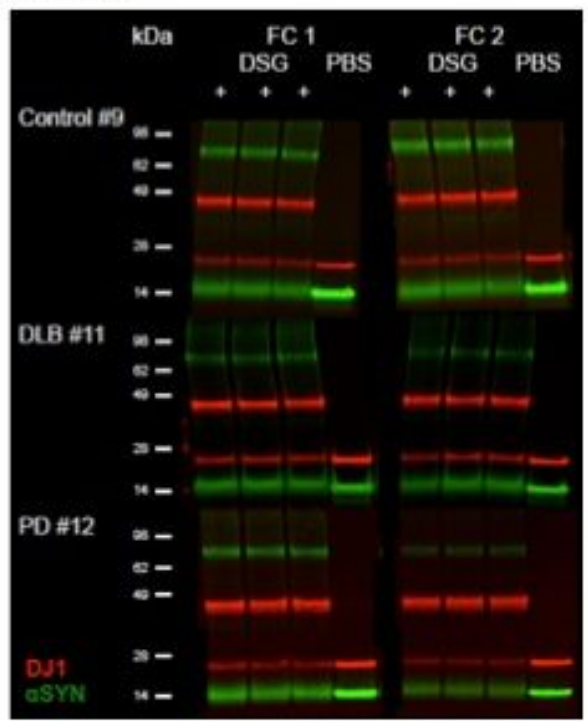

b CD spectra

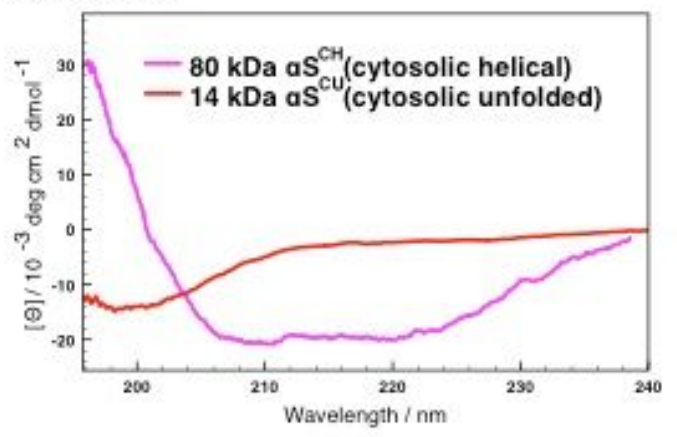

c Ratio $\alpha \mathrm{S}^{\mathrm{CH}} / \alpha \mathrm{S}^{\mathrm{cu}}$, cingulate cortex

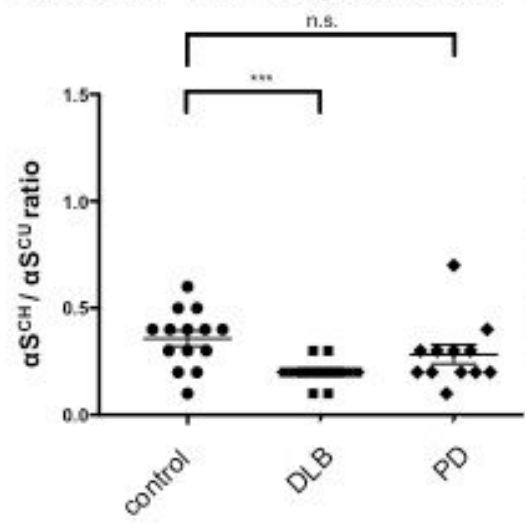

e Ratio $\alpha \mathrm{S}^{\mathrm{CH}} / \alpha \mathrm{S}^{\mathrm{CU}}$, entorhinal cortex

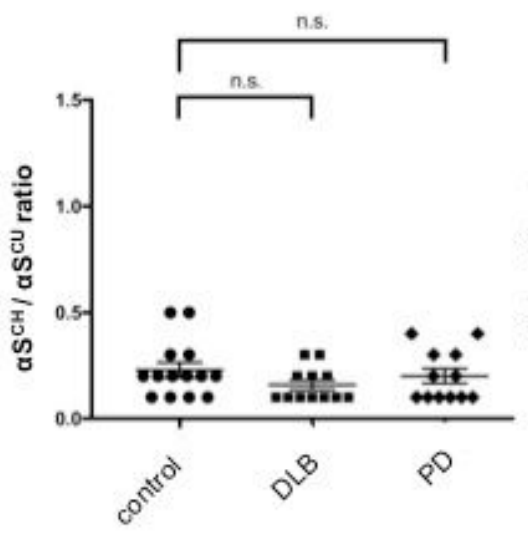

d Ratio $\alpha S^{\mathrm{CH} /} \alpha \mathrm{S}^{\mathrm{cU}}$, frontal cortex

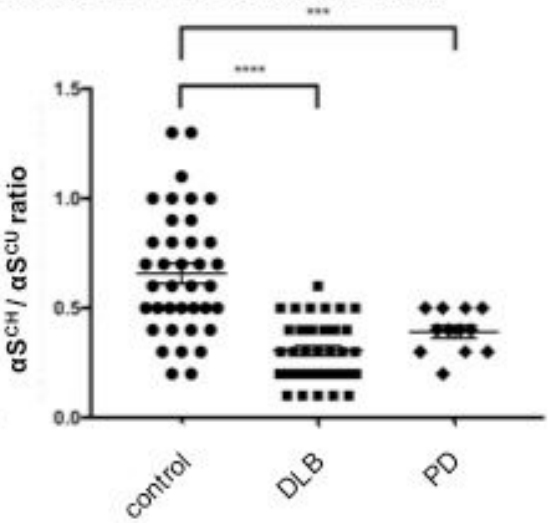

f Ratio $a S^{\mathrm{CH}} / \alpha \mathrm{S}^{\mathrm{CU}}$, striatum

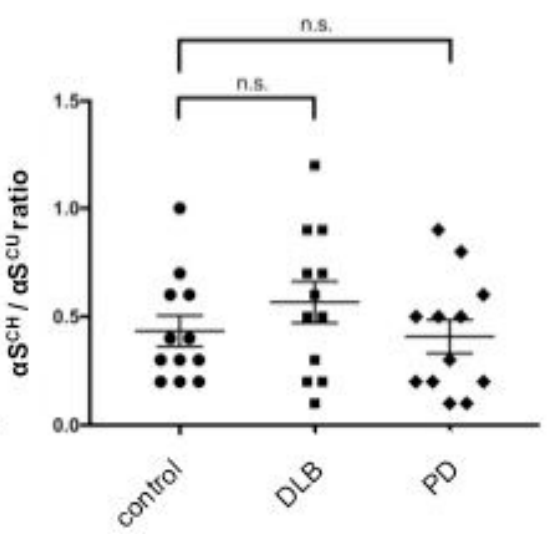

Figure 1

Disturbed equilibria of cytosolic helically folded and unfolded physiological aS in PD and DLB patients. a, Representative Western blot of aSCH and aSCU in controls, DLB and PD patients. Each piece of brain was analyzed in duplicates (frontal cortex FC1 and FC2). The crosslinking reaction was performed in technical triplicates alongside one non-crosslinked (PBS) sample. The Western blot demonstrates reduced aSCH to aSCU ratios in DLB and PD patients compared to the control. DSG " + " = crosslinked sample. DSG "-" = non-crosslinked sample. Green = aS, red = DJ1. b, CD spectroscopy of immunoprecipitated and separated (size-exclusion chromatography) aSCH and aSCU from human frontal cortex control brain tissue. The aS multimer from human brain exhibits an a-helical secondary structure of approximately $48 \%$. c, Significant reduction of aSCH/aSCU ratio in the cingulate cortex comparing controls $(n=7)$ and DLB $(n=$ 7) patients. No significant alteration of the aSCH/aSCU equilibria in the cingulate cortex comparing controls $(n=7)$ and PD $(n=6)$ patients. d, Significant reduction of aSCH/aSCU ratio in the frontal cortex comparing controls $(n=19)$ to PD $(n=6)$ and DLB $(n=19)$ patients. e, No significant alteration of the 
aSCH/aSCU equilibria in the entorhinal cortex comparing controls $(n=7)$ to PD $(n=6)$ and DLB $(n=6)$ patients. $f$, No significant alteration of the aSCH/aSCU equilibria in the striatum comparing controls $(n=$ 6) to PD $(n=6)$ and DLB $(n=6)$ patients.
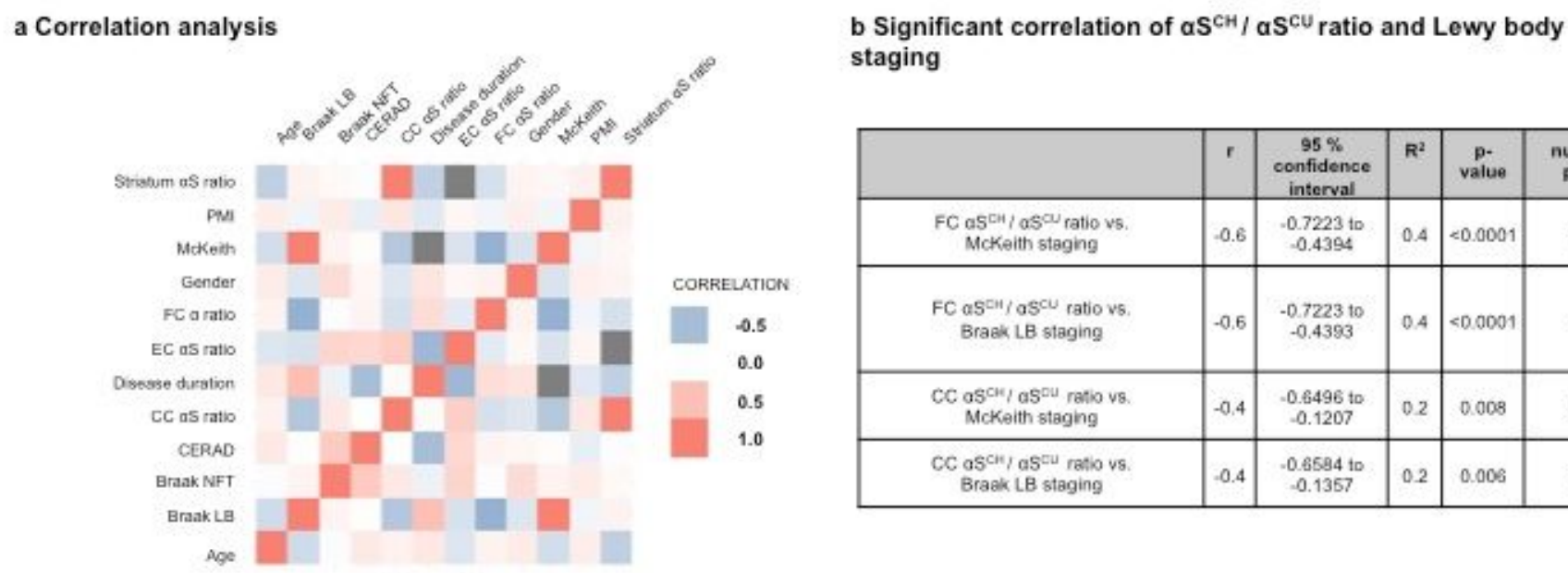

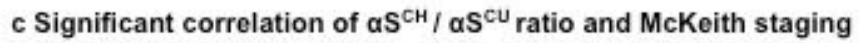

\begin{tabular}{|c|c|c|c|c|c|}
\hline & $r$ & $\begin{array}{c}95 \% \\
\text { confidence } \\
\text { interval }\end{array}$ & $\mathrm{R}^{2}$ & $\begin{array}{c}\text { p- } \\
\text { value }\end{array}$ & $\begin{array}{c}\text { number } \\
\text { pairs }\end{array}$ \\
\hline $\begin{array}{l}\text { FC } \mathrm{aS}^{\mathrm{CH}} \text { / } \alpha \mathrm{S}^{\mathrm{Cu}} \text { ratio vs. } \\
\text { Mckeith staging }\end{array}$ & -0.6 & $\begin{array}{c}-0.7223 \text { to } \\
-0.4394\end{array}$ & 0.4 & $<0.0001$ & 82 \\
\hline 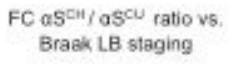 & -0.6 & $\begin{array}{c}-0.7223 \text { to } \\
-0.4393\end{array}$ & 0.4 & $<0,0001$ & 82 \\
\hline $\begin{array}{c}\mathrm{CC} \mathrm{aS}^{\mathrm{CH}} / \mathrm{aS}^{\mathrm{Du}} \text { ratio vs. } \\
\text { McKeith staging }\end{array}$ & -0.4 & $\begin{array}{l}-0.6496 \text { to } \\
-0.1207\end{array}$ & 0.2 & 0.008 & 39 \\
\hline 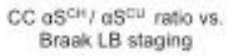 & -0.4 & $\begin{array}{l}-0.6584 \text { to } \\
-0.1357\end{array}$ & 0.2 & 0.006 & 39 \\
\hline
\end{tabular}
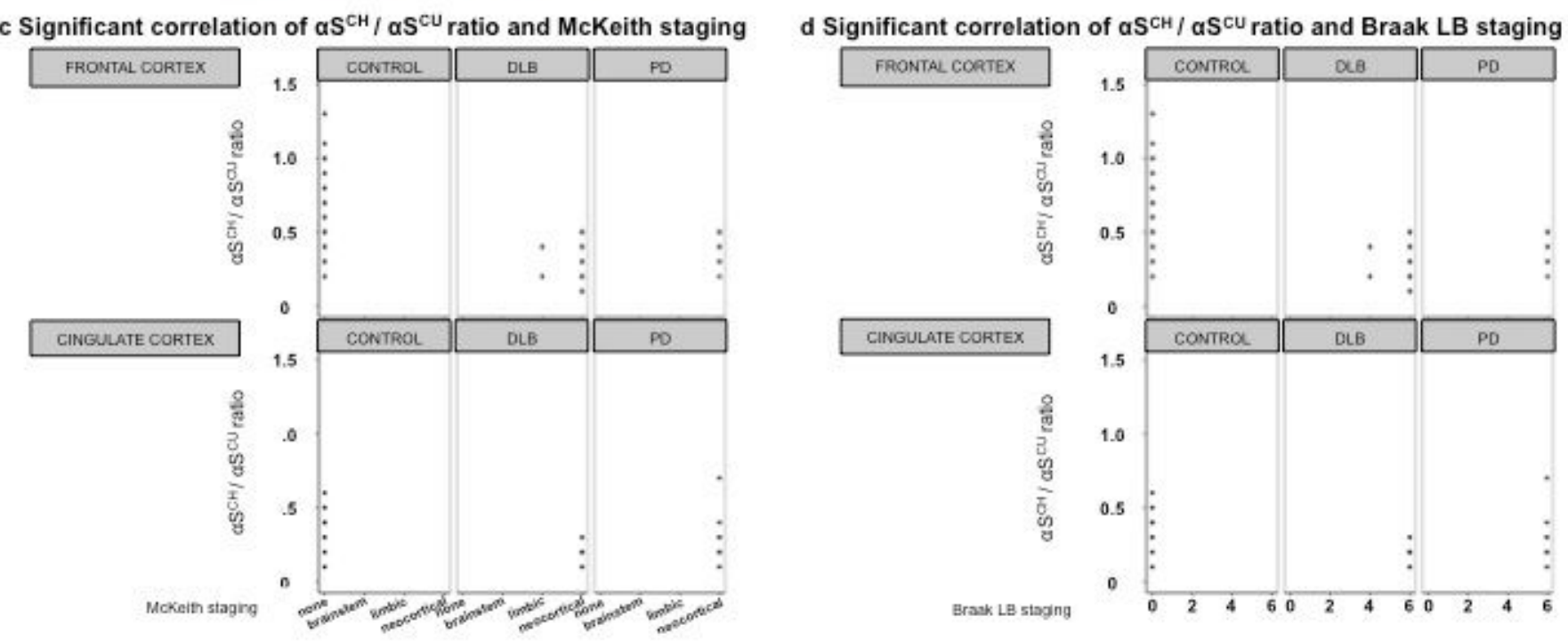

Figure 2

Decreased aSCH/aSCU ratios correlate with LB pathology. a, Heatmap of Pearson correlation coefficients. Controls were set to 0 for McKeith and Braak LB (LB) staging. b, Significant correlation (Pearson correlation coefficient) of decreased aSCH/aSCU ratios and McKeith or LB staging in the frontal and cingulate cortex, respectively. c, Graph of correlated samples (aSCH/aSCU ratios vs. McKeith). d, Graph of correlated samples (aSCH/aSCU vs. Braak LB). LB = Lewy body, NFT = neurofibrillary tangle, CERAD = Consortium to Establish a Registry for Alzheimer's Disease, $\mathrm{CC}=$ cingulate cortex, EC = entorhinal cortex, $\mathrm{FC}=$ frontal cortex, $\mathrm{PMI}=$ post-mortem interval. 


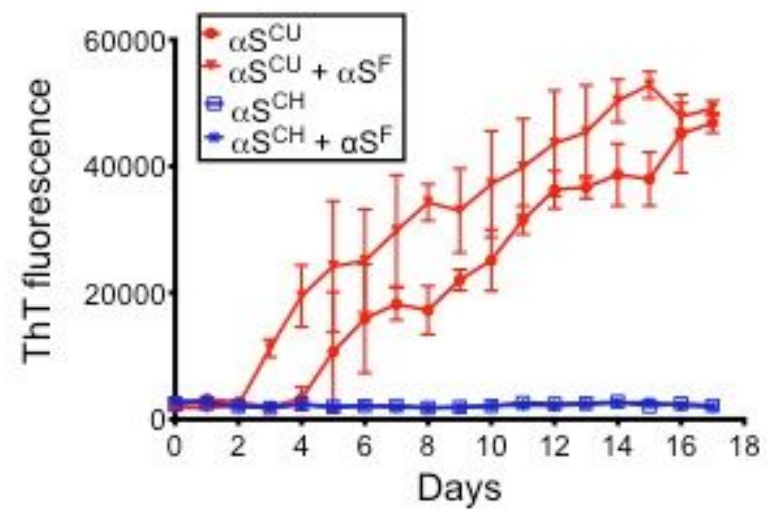

c Amounts of insouble aS in $\mathrm{aS}^{\mathrm{F}}$ seeded M17D fPD

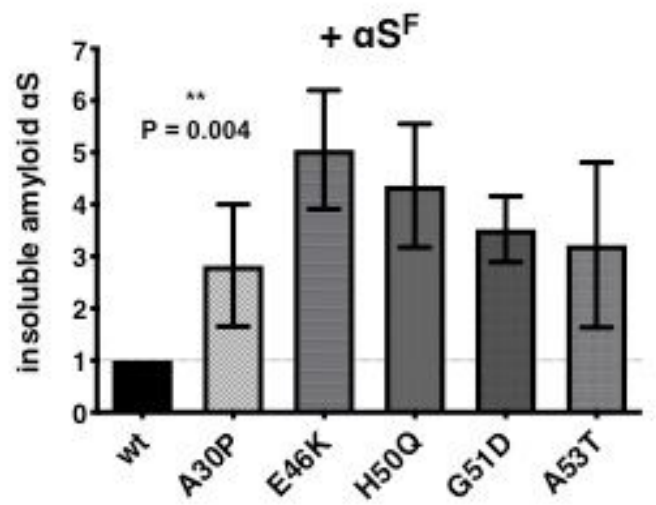

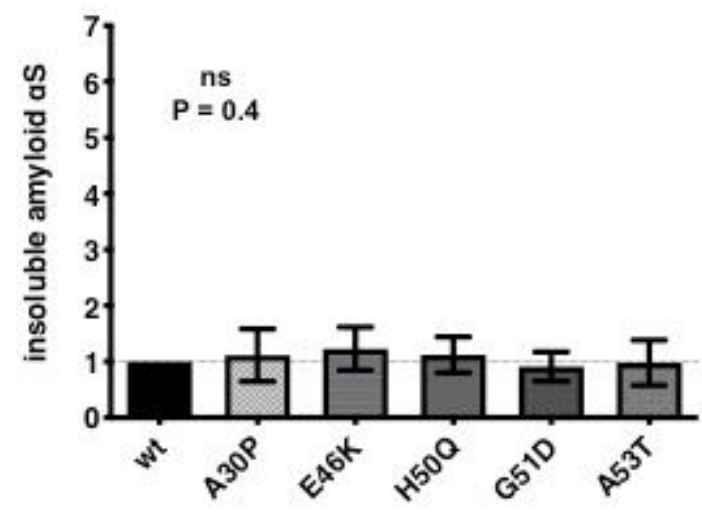

d Correlation of $\alpha \mathrm{S}^{\mathrm{CH} /} \alpha \mathrm{S}^{\mathrm{cu}}$ ratio and insoluble $\alpha \mathrm{S}$ in M17D fPD

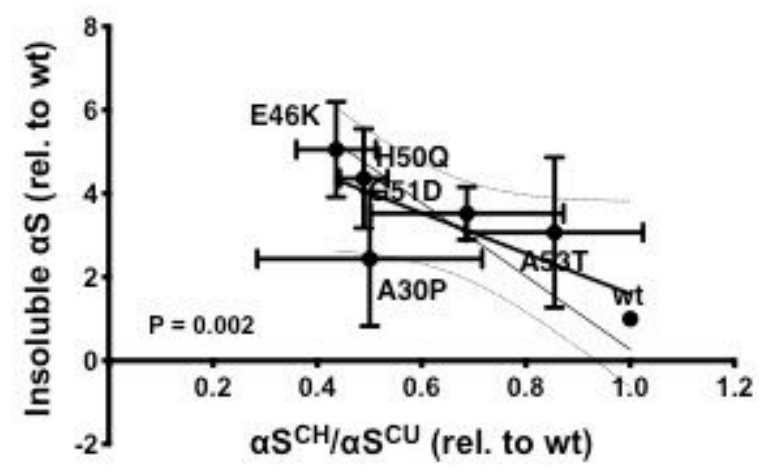

\section{Figure 3}

aSCH is resistant to spontaneous or "prion-like" induced aggregation compared to aSCU and modulates "prion-like" propagation susceptibility in cellular models of disease. a, Thioflavin T fluorescence assay monitoring the aggregation of recombinant aSCU and purified aSCH from erythrocytes. Samples were $100 \mu \mathrm{M}$ aS protein each, nutated at $37^{\circ} \mathrm{C}$. In samples that were seeded, $10 \mathrm{nM}$ recombinant fibrillar aS (aSF) (i.e., 1:1000) was added. The aSCH multimer demonstrated resistance to both spontaneous and seeded aggregation. b, M17D cells transfected with wild type aS, or the fPD related mutations A30P, E46K, H50Q, G51D and A53T. Cells with fPD mutations display equal amounts of insoluble aS. c, aSF-seeded M17D cells display increased levels of insoluble aS. $d$, aSCH destabilization correlates significantly (Deeming regression analysis) with susceptibility towards aS aggregation as measured by insoluble aS in M17D after 2 days of seeding $(P=0.002)$. Cells were analyzed in biological triplicates. 

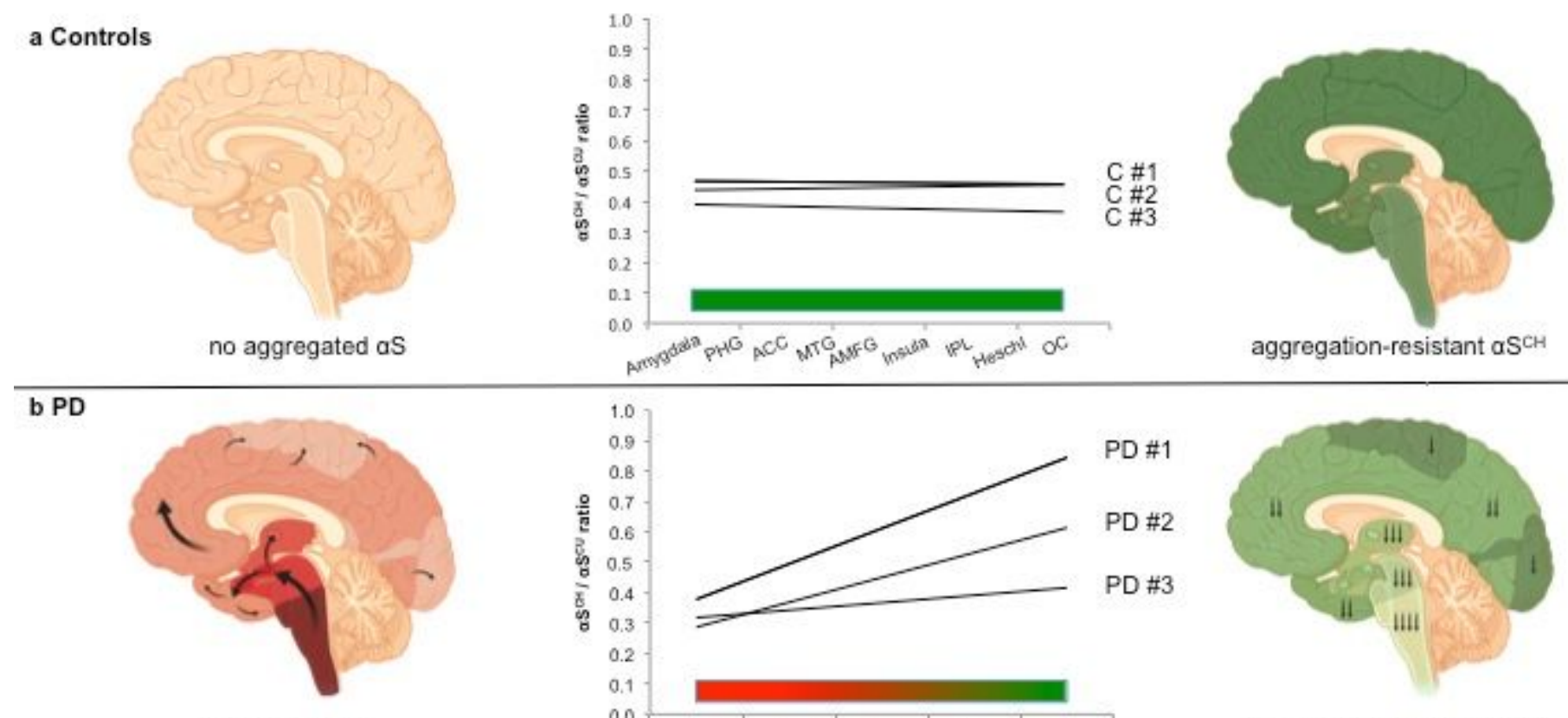

aggregated aS
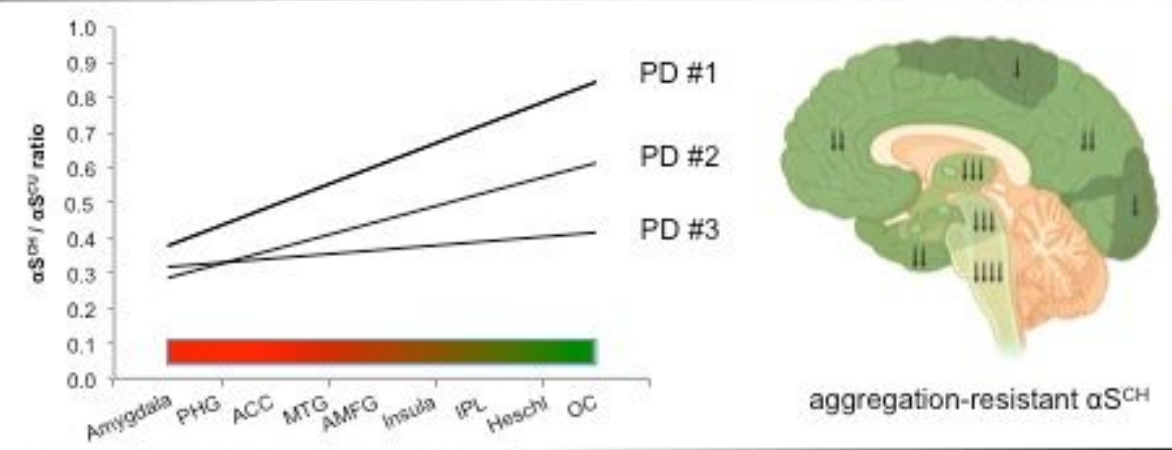

aggregation-resistant $\alpha \mathrm{S}^{\mathrm{CH}}$

\section{c DLB}
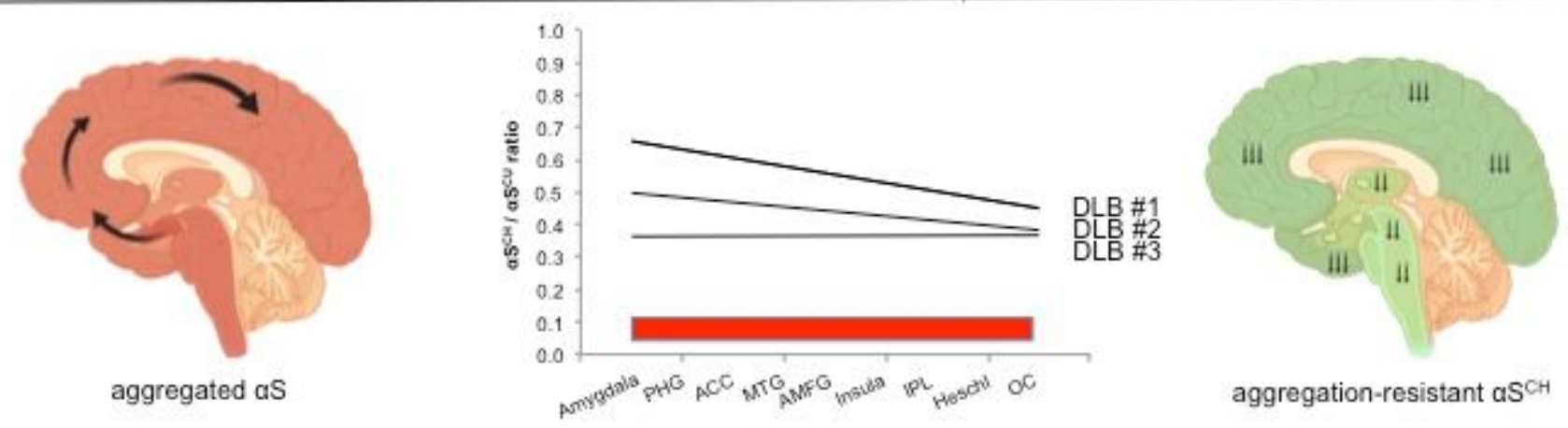

\section{Figure 4}

aSCH/aSCU equilibrium is disturbed in PD and DLB patients suggestive of spreading aS pathology. For each individual, 9 different brain regions were analyzed, reflecting the temporal development of LB pathology across the limbic and neocortical regions. Amygdala, cortex of the parahippocampal (PHG) and anterior cingulate gyri (ACG) are affected earlier in the disease course, followed by cortex of the insula, middle temporal (MTG), anterior middle frontal gyri (AMFG), and lastly with involvement of the cortex of inferior parietal lobule (IPL). Heschl's gyrus (Heschl) and cortex of the occipital lobe (OC) are typically spared from LB pathology in PD or involved late in the disease course. Each brain region has been analyzed in biological and technical duplicates and one non-crosslinked control sample. The linear trendlines (slopes) across all 9 brain regions is depicted for each individual. a, In controls, there is a balanced level of physiological aSCH/aSCU. b, PD patients (Braak 6) exhibit lower aSCH/aSCU ratios in early affected regions according to the classical Braak staging. C, DLB patients exhibit especially low aSCH/aSCU ratios in later affected, neocortical regions, including Heschl's gyrus and OC. Note that the relative measured aSCH/aSCU levels, can be lower in individual control cases compared to patients even though the difference is on average reversed. The schematics on the left and right side describe the proposed model for the disequilibria of aggregated aS and physiological, aggregation-resistant aSCH. 


\section{Supplementary Files}

This is a list of supplementary files associated with this preprint. Click to download.

- SupplementarymaterialdeBonietal.brainaSmultimers.pdf 